\title{
CALCULATION OF MANIN'S INVARIANT FOR DEL PEZZO SURFACES
}

\author{
TOHSUKE URABE
}

\begin{abstract}
For $r=7$ and 8 we consider an action of the Weyl group of type $E_{r}$ on a unimodular lattice of rank $r+1$. We give the tables of the first cohomology groups for all cyclic subgroups of the Weyl group with respect to this action. These are important in the arithmetic theory of Del Pezzo surfaces.
\end{abstract}

\section{INTRODUCTION}

In his book "Cubic Forms" [11] Manin proposed several ideas related to the Brauer group. (The Brauer equivalence, a method for giving counterexamples to the Hasse principle, etc.) Let $Y$ be a surface defined over a perfect field $k$, and $\bar{k}$ be the algebraic closure of $k$. We assume that the pull-back $X=Y \otimes \bar{k}$ is a projective irreducible smooth surface over $\bar{k}$. Manin considered the Brauer group $\operatorname{Br}(Y)$ (Milne [13, Chapter IV]). This is very useful in the arithmetic of surfaces. (See also Swinnerton-Dyer [14].)

In [11] Manin showed also several examples of cubic surfaces in three-dimensional projective space $\mathbf{P}^{3}$ among others, and suggested the extension of his theory to general Del Pezzo surfaces. Recall that $X$ is called a Del Pezzo surface, if the anticanonical line bundle $-\omega$ of $X$ is ample. It is known that for Del Pezzo surfaces the degree $d=(\omega, \omega)$ satisfies $1 \leq d \leq 9$. A Del Pezzo surface of degree 3 is nothing but a cubic surface in $\mathbf{P}^{3}$. In this article we give tables of some data for Del Pezzo surfaces of degrees 2 and 1. The Supplement section at the end of this issue contains our main tables. Our tables correspond to Manin's table of [11, pp. 176-177] in the case of degree 3.

In order to apply Manin's idea to concrete examples, we would like to determine $\operatorname{Br}(Y)=H^{2}\left(Y_{e t}, \mathbb{G}_{m}\right)$ explicitly. For this we can use the HochschildSerre spectral sequence $E_{2}^{p, q}=H^{p}\left(\mathrm{Gal}, H^{q}\left(X_{e t}, \mathbb{G}_{m}\right)\right) \Rightarrow H^{p+q}\left(Y_{e t}, \mathbb{G}_{m}\right)$, where $\mathrm{Gal}=\operatorname{Gal}(\bar{k} / k)$ is the Galois group of the extension $\bar{k} / k$. Note here that $E_{2}^{2,0}=$ $H^{2}\left(\mathrm{Gal}, \bar{k}^{*}\right)$ coincides with $\operatorname{Br}(k)$ by definition, and does not depend on the geometry of $Y$ and $X$ : it is not very interesting. Also, $E_{2}^{1,1}=H^{1}(\operatorname{Gal}, \operatorname{Pic}(X))$, where $\operatorname{Pic}(X)=H^{1}\left(X_{e t}, \mathbb{G}_{m}\right)$ denotes the Picard group of $X$.

Any Del Pezzo surface is birationally equivalent to the two-dimensional projective space $\mathbf{P}^{2}$ and has the following properties: first, since $H^{2}\left(X_{e t}, \mathbb{G}_{m}\right)=0$ (Milne [12]), we have $E_{2}^{0,2}=0$, and thus $\operatorname{Br}(Y) / \operatorname{Br}(k) \cong \operatorname{Ker}\left(H^{1}(\operatorname{Gal}, \operatorname{Pic}(X))\right.$ $\left.\rightarrow H^{3}\left(\mathrm{Gal}, \bar{k}^{*}\right)\right)$. Second, $\operatorname{Pic}(X)$ is a discrete group and coincides with the Neron-

Received by the editor November 23, 1993 and, in revised form, February 22, 1995.

1991 Mathematics Subject Classification. Primary 20J06; Secondary 13A20.

Key words and phrases. Brauer group, Del Pezzo surface, Weyl group. 
Severi group $\operatorname{NS}(X)$ of $X$. Third, $\operatorname{NS}(X)$ is a free $\mathbf{Z}$-module of rank $10-d$. Fourth, the intersection form is a symmetric bilinear form $(\cdot, \cdot): \mathrm{NS}(X) \times \mathrm{NS}(X) \rightarrow \mathbf{Z}$ and it defines the structure of a lattice with discriminant 1 and signature $(1,9-d)$ on $\mathrm{NS}(X)$.

Following Manin, we assume moreover that the image $G$ of the homomorphism $\mathrm{Gal} \rightarrow$ Aut $(\mathrm{NS}(X))$ is a cyclic group. Under this assumption we have $\operatorname{Br}(Y) / \operatorname{Br}(k)$ $\cong H^{1}(\operatorname{Gal}, \operatorname{Pic}(X)) \cong H^{1}(G, \mathrm{NS}(X))$. Therefore, the calculation of the group $H^{1}(G, \operatorname{NS}(X))$ has great importance.

Now, by lattice theory, the structure of the lattice $\mathrm{NS}(X)$ is uniquely determined by the degree $d$, if $d \neq 8$. (When $d=8$ there are two possibilities.) Therefore, we can denote it by $U_{r}=\mathrm{NS}(X)$ with $r=9-d$ if $1 \leq d \leq 7$. The lattice $U_{r}$ is the unimodular lattice with signature $(1, r)$ and is defined for $2 \leq r \leq 8$. Let $W\left(R_{r}\right)$ be the group of automorphisms of $U_{r}$ preserving the intersection form and fixing the canonical class $\omega \in U_{r}$. Note that the image $G$ of the Galois group Gal is contained in $W\left(R_{r}\right)$. It is known that for $3 \leq r \leq 8$ the group $W\left(R_{r}\right)$ is a finite group isomorphic to a Weyl group appearing in the theory of Lie groups (Bourbaki [1, Ch. VI]), and its action on the orthogonal complement of $\omega \in U_{r}$ can be identified with the action of the Weyl group on the Cartan subalgebra of a semisimple Lie algebra. The type of $W\left(R_{r}\right)$ is $A_{2}+A_{1}$ (when $\left.r=3\right), A_{4}(r=4), D_{5}(r=5), E_{6}$ $(r=6), E_{7}(r=7)$ and $E_{8}(r=8)$ respectively. (The discovery of the connection between Weyl groups and Del Pezzo surfaces is very old. For example, see Cartan [3], Du Val [6]. For a modern treatment see Manin [11, p. 129, Theorem 25.4].)

We find that our problem is the following: calculate the group $H^{1}\left(G, U_{r}\right)$ for every conjugacy class of $W\left(R_{r}\right)$, where $G$ is the cyclic group generated by an element belonging to the conjugacy class.

When $r \leq 6$, the results have been given in [11] subject to some necessary corrections that we shall discuss below. (See also Kunyavskii, Skorobogatov and Tsfasman [9].) In particular, we can find a table of $H^{1}\left(G, U_{r}\right)$ for $r=5,6$ in [11]. In this article we give tables of conjugacy classes and their associated groups $H^{1}\left(G, U_{r}\right)$ for $r=7$ and $r=8$. For the classification of conjugacy classes in the Weyl group of type $E_{7}$ or $E_{8}$ we have the results of Carter [4]. We apply his results.

Note that a Del Pezzo surface of degree 2 corresponding to $r=7$ is a smooth surface in the weighted projective space $\mathbf{P}(2,1,1,1)$ defined by a polynomial $w^{2}+F_{4}(x, y, z)$, where $F_{4}$ is a homogeneous polynomial of degree 4 , and a Del Pezzo surface of degree 1 corresponding to $r=8$ is a smooth surface in the weighted projective space $\mathbf{P}(3,2,1,1)$ defined by a polynomial $w^{2}+z^{3}+G_{4}(x, y) z+G_{6}(x, y)$, where $G_{4}, G_{6}$ are homogeneous polynomials of degree 4 and 6 , respectively. The connection to the arithmetic of curves of genus 3 (the plane curve defined by $F_{4}(x, y, z)$ is the canonical model of a curve of genus 3$)$ and to the arithmetic of elliptic curves (for general constants $a, b$ the polynomial $w^{2}+z^{3}+G_{4}(a, b) z+G_{6}(a, b)$ defines an elliptic curve) may be very interesting. Moreover, since the group $W\left(R_{r}\right)$ is isomorphic to the Weyl group of type $E_{r}$ for $r=6,7,8$, the connection to Weyl groups may also be interesting. This article is the starting point of a study of the case of degree 2 and 1. (See [11, p. 115, Remark 23.8.1]. Besides, there may be a connection to Lie groups. It is quite natural to expect that behind Weyl groups there are hidden Lie groups. Of course, no reasonable connections between Lie groups and Del Pezzo surfaces are known at present. It would be interesting to discover this connection, if it exists.) 
In $\S 1$ we try to give a clear theoretical description of the structure of the group $H^{1}\left(G, U_{r}\right)$ as a preparation. Among other things, we show that $H^{1}\left(G, U_{r}\right)$ carries a nondegenerate alternating bilinear form with values in $\mathbf{Q} / \mathbf{Z}$. Thus in particular, the order $h^{1}$ of $H^{1}\left(G, U_{r}\right)$ is always a square number.

Therefore, it follows that the seventh column in Manin's table for the case $d=3$, $r=6$ on page 176 in [11] contains two mistakes. The seventh item $\mathbf{Z}_{2}$ corresponding to the conjugacy class $C_{20}$, and the tenth item $\mathbf{Z}_{2}$ corresponding to $C_{4}$, have to be replaced by 0 , since the order of $\mathbf{Z}_{2}$ is not a square number. Also the table for the case $d=4, r=5$ on page 178 in [11] or on page 454 in [10] contains two mistakes at the items corresponding to $C_{20}$ and $C_{4}$ in the case $d=3, r=6$. Related to these mistakes of Manin, a result in Milne [12] can be improved: the Brauer group of a rational surface over a finite field always carries a canonical nondegenerate alternating form. Milne did not try to show that the bilinear form is alternating, because he had believed Manin's false data which contradicts the alternating property.

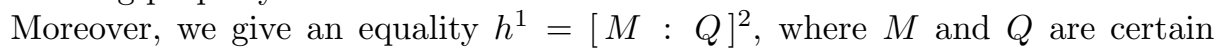
submodules in $U_{r}$. Using this equality, we can determine $h^{1}$.

It turns out that the theory developed here gives an effective method for determining the group $H^{1}\left(G, U_{r}\right)$. Except for a few $E_{8}$ cases, the calculation is easy. However, the number of conjugacy classes is quite large.

Section 2 is devoted to the explanation of our main tables. The tables are given in the Supplement section at the end of this issue. Now, for the application to concrete examples we need to determine to which conjugacy class of the Weyl group the given cyclic action on $U_{r}$ of the Galois group corresponds. For this purpose we have added the value of the index and the information on the orbit decomposition of the exceptional set to each conjugacy class in our tables. The index and the orbit decomposition were calculated using the "Mathematica" software running on a Macintosh II cx personal computer (Wolfram [16]). To collect data, we used the software "Excel".

I have put the Mathematica notebooks used in my calculation in the anonymous ftp server at my university (ftp.math.metro-u.ac.jp. IP address 133.86.76.25. Urabe [15]). The reader can check the data in this paper using these notebooks after getting them by anonymous ftp.

A free $\mathbf{Z}$-module of finite rank equipped with a symmetric bilinear form $(\cdot, \cdot)$ with values in $\mathbf{Z}$ is called a lattice. The discriminant $\operatorname{det} L$ of a lattice $L$ is defined by det $L=\left|\operatorname{det}\left(e_{i}, e_{j}\right)\right|$, where $e_{1}, e_{2}, \ldots, e_{r}$ is a basis of $L$. (det $L$ is independent of the choice of the basis $e_{1}, e_{2}, \ldots, e_{r}$.) If det $L=1$, then $L$ is called unimodular. If det $L \neq 0$, then $L$ is called nondegenerate. A cyclic group of order $k$ is denoted by $\mathbf{Z}_{k}$. When a group $G$ acts on a set $S$, by $S^{G}=\{x \in S \mid g(x)=x$ for every $g \in G\}$ we denote the set of fixed elements. If $G$ is a cyclic group of order $m$ with a generator $w \in G$ and if $S$ is a $G$-module, we consider two special elements $T=1-w$ and $N=\sum_{k=0}^{m-1} w^{k}$ in particular. The homomorphisms $T, N: S \rightarrow S$ induced by them are denoted by the same symbols. By definition $T(x)=(1-w) x=x-w(x)$ and $N(x)=\left(\sum_{k=0}^{m-1} w^{k}\right) x=\sum_{k=0}^{m-1} w^{k}(x)$. In this case, $S^{G}=\operatorname{Ker}(T: S \rightarrow S)$.

Remark. Related to the order $h^{1}$ of $H^{1}\left(G, U_{r}\right)$ there is a conjecture by Manin. Denote by $(\varsigma)$ the set of characteristic roots different from 1 of a generator of the 
cyclic group $G$ acting on $U_{r}$, and by $\Delta$ the discriminant of the sublattice $U_{r}^{G}$ of fixed elements. Then, Conjecture 31.7 on page 182 in [11] says, for any Del Pezzo surface $X$ of degree $d$ with $1 \leq d \leq 6$, that the equality $\prod_{\varsigma}(1-\varsigma)=h^{1} \Delta$ holds. This conjecture has already been confirmed by Milne [12] and Zarkhin [17], and this provides another method for calculating $h^{1}$. (However, it does not follow from it that $h^{1}$ is a square number.)

\section{Action of a CyClic group on a lattice}

Let $L$ be a nondegenerate lattice and $G$ be a finite cyclic group of order $m$ acting on $L$. By $w$ we denote a generator of $G$. Assume that the action of $G$ on $L$ preserves the bilinear form.

Let $G^{\vee}=\{\chi \mid \chi: G \rightarrow \mathbf{C}-\{0\}$ is a group homomorphism $\}$ denote the group of characters of $G$. Set $(L \otimes \mathbf{C})_{\chi}=\{x \in L \otimes \mathbf{C} \mid g(x)=\chi(g) x$ for every $g \in G\}$ for $\chi \in G^{\vee}$.

Lemma 1.1. There holds $L \otimes \mathbf{C}=\sum_{\chi}(L \otimes \mathbf{C})_{\chi}$ (a direct sum).

The natural $\mathbf{C}$-bilinear extension of $(\cdot, \cdot)$ on $L \otimes \mathbf{C}$ is denoted by the same symbol $(\cdot, \cdot)$. A Hermitian bilinear form $((\cdot, \cdot))$ on $L \otimes \mathbf{C}$ is defined by $((x, y))=(x, \bar{y})$ for $x, y \in L \otimes \mathbf{C}$ where $\bar{y}$ denotes the complex conjugate of $y$.

Lemma 1.2. The direct sum in Lemma 1.1 is an orthogonal direct sum with respect to the Hermitian form $((\cdot, \cdot))$.

Lemma 1.3. If $y \in(L \otimes \mathbf{C})^{G}$, then $\bar{y} \in(L \otimes \mathbf{C})^{G}$.

By $\varepsilon \in G^{\vee}$ we denote the identity character. By definition $(L \otimes \mathbf{C})_{\varepsilon}=(L \otimes \mathbf{C})^{G}=$ $\operatorname{Ker}(T: L \otimes \mathbf{C} \rightarrow L \otimes \mathbf{C})$. Set $\Gamma=\operatorname{Ker}(N: L \otimes \mathbf{C} \rightarrow L \otimes \mathbf{C})$. Obviously $\Gamma=\sum_{\chi \neq \varepsilon}(L \otimes \mathbf{C})_{\chi}$. Let $M$ be the orthogonal complement of $L^{G}$ in $L$.

Lemma 1.4. We have $M=\operatorname{Ker}(N: L \rightarrow L)$.

Proof. By Lemma 1.2, $\Gamma$ and $(L \otimes \mathbf{C})^{G}$ are orthogonal with respect to the Hermitian form $((\cdot, \cdot))$. By Lemma 1.3 they are orthogonal also with respect to the $\mathbf{C}$-bilinear form $(\cdot, \cdot)$. Since $(\cdot, \cdot)$ is nondegenerate by assumption, by Lemma $1.1, \Gamma$ is the orthogonal complement of $(L \otimes \mathbf{C})^{G}=(L \otimes \mathbf{C})_{\varepsilon}$. This implies the lemma.

Corollary 1.5. Sublattices $L^{G}$ and $M$ are nondegenerate.

Proof. By Lemmas 1.4 and 1.1 we have $L^{G} \cap M \subset(L \otimes \mathbf{C})^{G} \cap \Gamma=0$.

Lemma 1.6. We have $T(L) \subset M$ and $H^{1}(G, L) \cong M / T(L)$.

Proof. We consider $H^{1}(G, L) \cong \operatorname{Ext}_{\mathbf{Z}[G]}^{1}(\mathbf{Z}, L)$, where $\mathbf{Z}[G]$ is the group ring of $G$. We have the following $\mathbf{Z}[G]$-free resolution of the $\mathbf{Z}[G]$-module $\mathbf{Z}$ :

$$
0 \leftarrow \mathbf{Z} \leftarrow \mathbf{Z}[G] \stackrel{T}{\longleftarrow} \mathbf{Z}[G] \stackrel{N}{\longleftarrow} \mathbf{Z}[G] \stackrel{T}{\longleftarrow} \mathbf{Z}[G] \leftarrow \cdots .
$$

Since $L \cong \operatorname{Hom}_{\mathbf{Z}[G]}(\mathbf{Z}[G], L)$, we have the induced sequence

$$
L \stackrel{T}{\longrightarrow} L \stackrel{N}{\longrightarrow} L \stackrel{T}{\longrightarrow} L \rightarrow \cdots .
$$


By Lemma 1.4 we have

$$
H^{1}(G, L) \cong \operatorname{Ext}_{\mathbf{Z}[G]}^{1}(\mathbf{Z}, L)=\operatorname{Ker}(N: L \rightarrow L) / \operatorname{Im}(T: L \rightarrow L)=M / T(L) .
$$

For a nondegenerate lattice $K, K^{*}=\{x \in K \otimes \mathbf{Q} \mid(x, y) \in \mathbf{Z}$ for every $y \in K\}$ is called the dual module of $K$. The natural $\mathbf{Q}$-valued extension on $K \otimes \mathbf{Q}$ of the bilinear form on $K$ is denoted by the same symbol $(\cdot, \cdot)$ here. Obviously $K^{*} \supset K$ and their indexes satisfy $\left[K^{*}: K\right]=\operatorname{det} K$. If $K$ has an action of a group $G$, then the action is extended to $K^{*}$.

Note here that $T: L \rightarrow M$ is the composition of the canonical surjective homomorphism $L \rightarrow L / L^{G}$ and an injective homomorphism $\bar{T}: L / L^{G} \rightarrow M$. Now, since $\left(L / L^{G}\right) \otimes \mathbf{Q}$ can be identified with $M \otimes \mathbf{Q}, L / L^{G}$ is identified with a submodule $M^{\prime}$ in $M \otimes \mathbf{Q}$. Moreover, if $\xi \in L$ is a representative of an element $x \in M^{\prime}=L / L^{G}$, then $(x, y)=(\xi, y) \in \mathbf{Z}$ for every $y \in M$. Thus, one has $M \subset M^{\prime} \subset M^{*}$. Under this identification, $\bar{T}$ above is identified with the restriction of $T: M^{*} \rightarrow M^{*}$ to $M^{\prime}$.

Corollary 1.7. The order $h^{1}$ of $H^{1}(G, L)$ is finite.

Proof. Since $T: M \rightarrow M$ is injective and since $M$ is a free $\mathbf{Z}$-module of finite rank, the index $[M: T(M)]$ is finite. Thus $h^{1}=\left[M: T\left(M^{\prime}\right)\right] \leq[M: T(M)]$ is also finite.

Lemma 1.8. Assume that $K$ and $M$ are nondegenerate sublattices of a unimodular lattice $U$. Assume moreover that they are the orthogonal complements of each other. Then the image $M^{\prime}$ of the canonical homomorphism $U / K \rightarrow M \otimes \mathbf{Q}$ coincides with $M^{*}$.

Proof. Above we have shown $M^{\prime} \subset M^{*}$. Conversely, pick $x \in M^{*}$. Since $U$ is unimodular, we have an element $\xi \in U$ with $(x, y)=(\xi, y)$ for every $y \in M$. Let $x^{\prime} \in M^{\prime}$ be the image of $\xi$ under $U \rightarrow U / K \rightarrow M^{\prime}$. Then we have $\left(x-x^{\prime}, y\right)=0$ for every $y \in M$. We have $x=x^{\prime} \in M^{\prime}$, since $M$ is nondegenerate.

Applying Lemma 1.8 to $U=U_{r}$ and $K=U^{G}$, we have the following proposition.

Proposition 1.9. For any cyclic subgroup $G$ of $W\left(R_{r}\right)$ with a generator $w$ one has $H^{1}\left(G, U_{r}\right) \cong M / T\left(M^{*}\right)=M /(1-w) M^{*}$, where $M$ denotes the orthogonal complement of $U_{r}^{G}$ in $U_{r}$.

A bilinear form $\langle\cdot, \cdot\rangle: A \times A \rightarrow B$ over a finite abelian group $A$ with values in an abelian group $B$ is called skew-symmetric if $\langle x, y\rangle=-\langle y, x\rangle$ for every $x, y \in A$. A skew-symmetric bilinear form such that $\langle x, x\rangle=0$ for every $x \in A$ is called alternating. If the condition $\langle x, y\rangle=0$ for every $y \in A$ implies $x=0$, then the skew-symmetric bilinear form is called nondegenerate.

Now, the product $H^{1}(G, L) \times H^{1}(G, L) \rightarrow H^{2}(G, L \otimes L)$ is defined. The symmetric bilinear form on $L$ induces a homomorphism $H^{2}(G, L \otimes L) \rightarrow H^{2}(G, \mathbf{Z}) \cong$ $\mathbf{Z} / N(\mathbf{Z})=\mathbf{Z}_{m}$. (Here, $m$ is the order of the cyclic group $G$.) Thus we get a bilinear form $\langle\cdot, \cdot\rangle: H^{1}(G, L) \times H^{1}(G, L) \rightarrow H^{2}(G, \mathbf{Z})$. By cohomology theory this form is skew-symmetric.

If we fix an embedding of groups $H^{2}(G, \mathbf{Z}) \subset \mathbf{Q} / \mathbf{Z}$, we get a bilinear form $H^{1}(G, L) \times H^{1}(G, L) \rightarrow \mathbf{Q} / \mathbf{Z}$, which we denote by the same symbol $\langle\cdot, \cdot\rangle$.

The following theorem is essentially due to Zarkhin ([17, 3.4.1, Lemma b]). 
Theorem 1.10. Under the same assumption as in Proposition 1.9 the bilinear form $\langle\cdot, \cdot\rangle: H^{1}\left(G, U_{r}\right) \times H^{1}\left(G, U_{r}\right) \rightarrow H^{2}(G, \mathbf{Z})$ is alternating and nondegenerate.

Proof. Since $U_{r}$ is unimodular, the form is nondegenerate (Milne [12]). We show that it is alternating. Assume that cohomology classes $\bar{x}, \bar{y} \in H^{1}\left(G, U_{r}\right) \cong$ $M / T\left(M^{*}\right)$ are represented by the elements $x, y \in M$. Then, it is known that their product in $H^{2}\left(G, U_{r} \otimes U_{r}\right)$ is represented by $\sum_{0 \leq a<b<m} w^{a}(x) \otimes w^{b}(y)$ (Cartan, and Eilenberg [2, p. 251]). Thus, $\langle\bar{x}, \bar{y}\rangle$ is equal to the class of $\sum_{0 \leq a<b<m}\left(w^{a}(x), w^{b}(y)\right)$ in $\mathbf{Z}_{m}$.

Now, note that our $U_{r}$ has geometric origin. In the Neron-Severi group of a surface with canonical class $\omega$, the sum $(\omega, \xi)+(\xi, \xi)$ is always an even integer for any element $\xi$. This is because for any line bundle $\Xi$ corresponding to the element $\xi, \chi(\Xi)-\chi\left(\mathcal{O}_{X}\right)=((\omega, \xi)-(\xi, \xi)) / 2$ is an integer by Riemann-Roch. In our case, $U_{r}$ is a model of a Neron-Severi group of a surface. Moreover, $\omega \in U_{r}^{G}$ and $(\omega, x)=0$ for every $x \in M$. Thus, $(x, x) / 2$ is an integer for $x \in M$.

Since $N(x)=0$ for $x \in M$, we have

$$
\begin{aligned}
\sum_{0 \leq a<b<m}\left(w^{a}(x), w^{b}(x)\right) \\
=\frac{1}{2}\left(\sum_{0 \leq a<b<m}\left(w^{a}(x), w^{b}(x)\right)+\sum_{0 \leq a<b<m}\left(w^{b}(x), w^{a}(x)\right)\right) \\
=\frac{1}{2}\left(\sum_{a=0}^{m-1} \sum_{b=0}^{m-1}\left(w^{a}(x), w^{b}(x)\right)-\sum_{a=0}^{m-1}\left(w^{a}(x), w^{a}(x)\right)\right) \\
=\frac{1}{2}((N(x), N(x))-m(x, x)) \\
=-m(x, x) / 2 .
\end{aligned}
$$

Thus one knows $\langle\bar{x}, \bar{x}\rangle=0$.

In the following, for a finite abelian group $B$, we denote by $B^{\vee}$ the dual abelian group $\operatorname{Hom}(B, \mathbf{Q} / \mathbf{Z})$. The groups $B$ and $B^{\vee}$ are isomorphic.

A subgroup $I$ of an abelian group $A$ with a bilinear form $\langle\cdot, \cdot \cdot: A \times A \rightarrow \mathbf{Q} / \mathbf{Z}$ is called isotropic, if $\langle x, y\rangle=0$ for every $x, y \in I$.

Lemma 1.11. Let $A$ be a finite abelian group carrying a nondegenerate alternating bilinear form $\langle\cdot, \cdot\rangle: A \times A \rightarrow \mathbf{Q} / \mathbf{Z}$.

1. The homomorphism $\varphi: A \rightarrow A^{\vee}$ defined by $\varphi(x)(y)=\langle x, y\rangle$ induces an isomorphism of groups.

2. Let $I \subset A$ be a maximal isotropic subgroup. The homomorphism $\varphi$ induces isomorphisms $\alpha: I \rightarrow(A / I)^{\vee}$ and $\beta: A / I \rightarrow I^{\vee}$. In particular the order of $A$ is equal to the square of the order of $I$.

3. There exists a maximal isotropic subgroup $I \subset A$ with the following property: there is an isomorphism of groups $\psi: I+I^{\vee} \rightarrow A$ such that the restriction of $\psi$ to $I$ is the identity mapping and $\langle\psi(x), \psi(y)\rangle=\langle\langle x, y\rangle\rangle$ for $x \in I$ and $y \in I^{\vee}$, where $\langle\langle\cdot, \cdot\rangle\rangle: I \times I^{\vee} \rightarrow \mathbf{Q} / \mathbf{Z}$ denotes the canonical perfect pairing and $I+I^{\vee}$ denotes the direct sum of the abelian groups $I$ and $I^{\vee}$. 
Proof. Easy.

Remark. The above statement (3) implies that the exact sequence

$$
0 \rightarrow I \rightarrow A \rightarrow A / I \rightarrow 0
$$

splits. But all maximal isotropic subgroups do not have this property. Let $p$ be a prime number and consider an abelian group $A=\mathbf{Z}_{p^{2}}+\mathbf{Z}_{p^{2}}$ with a nondegenerate alternating bilinear form satisfying $\left\langle x_{0}, y_{0}\right\rangle=1 / p^{2}+\mathbf{Z} \in \mathbf{Q} / \mathbf{Z}$, where $x_{0}$ and $y_{0}$ are generators of respective components $\mathbf{Z}_{p^{2}}$. Let $I_{1}$ be the subgroup generated by $x_{0}$, and $I_{2}$ be the subgroup generated by $p x_{0}$ and $p y_{0}$. We can check easily that both $I_{1}$ and $I_{2}$ are maximal isotropic subgroups. One has $I_{1} \cong \mathbf{Z}_{p^{2}}$ and $I_{1}$ has the property in (3). But, $I_{2} \cong \mathbf{Z}_{p}+\mathbf{Z}_{p}$ and for $I_{2}$ the above exact sequence does not split.

Corollary 1.12. For some abelian group I there is an isomorphism

$$
H^{1}\left(G, U_{r}\right) \cong I+I .
$$

For $2 \leq r \leq 8$ the model $U_{r}$ of the Neron-Severi group of a Del Pezzo surface of degree $9-r$ has a basis $e_{0}, e_{1}, \ldots, e_{r}$ with $\left(e_{0}, e_{0}\right)=+1,\left(e_{i}, e_{i}\right)=-1$ for $1 \leq$ $i \leq r$ and $\left(e_{i}, e_{j}\right)=0$ for $i \neq j$, where $(\cdot, \cdot)$ denotes the intersection form. The canonical class corresponds to $\omega=-3 e_{0}+e_{1}+\cdots+e_{r}$. The set of roots is defined by

$$
R_{r}=\left\{\alpha \in U_{r} \mid(\alpha, \alpha)=-2,(\alpha, \omega)=0\right\} .
$$

Each root $\alpha \in R_{r}$ defines an automorphism $s_{\alpha}: U_{r} \rightarrow U_{r}$ called a reflection. It satisfies $s_{\alpha}(x)=x+(x, \alpha) \alpha$ for $x \in U_{r}$. It is easy to check that it preserves the intersection form, fixes $\omega$, and is of order 2, i.e., $s_{\alpha}^{2}=$ the identity. The group $W\left(R_{r}\right)$ generated by all $s_{\alpha}$ 's coincides with the group of automorphisms of the lattice $U_{r}$ preserving $\omega$ and the intersection form ([11, p. 115, Theorem 23.9]).

Remark. For $3 \leq r \leq 8, R_{r}$ generates the orthogonal complement of $\omega$ in $U_{r}$. In the case $r=2, R_{2}$ consists of only two elements $R_{2}=\left\{e_{1}-e_{2},-e_{1}+e_{2}\right\}$ and it never generates the orthogonal complement of $\omega$. However, even if $r=2$, $W\left(R_{2}\right) \cong \mathbf{Z}_{2}$ coincides with the group of automorphisms preserving $\omega$ and the intersection form.

Lemma 1.13 (Carter [4, Lemma 3, Lemma 2]). (1) For every $w \in W\left(R_{r}\right)$ there are linearly independent roots $\alpha_{1}, \alpha_{2}, \ldots, \alpha_{k} \in R_{r}$ with $w=s_{\alpha_{1}} s_{\alpha_{2}} \cdots s_{\alpha_{k}}$.

(2) If $\alpha_{1}, \alpha_{2}, \ldots, \alpha_{k} \in R_{r}$ are linearly independent, then the sublattice $U_{r}^{w}$ of elements fixed by $w=s_{\alpha_{1}} s_{\alpha_{2}} \cdots s_{\alpha_{k}}$ coincides with the orthogonal complement of the sublattice generated by $\alpha_{1}, \alpha_{2}, \ldots, \alpha_{k} \in R_{r}$.

Let $G \subset W\left(R_{r}\right)$ be a cyclic subgroup and $w \in G$ be a generator. Let $\alpha_{1}, \alpha_{2}$, $\ldots, \alpha_{k} \in R_{r}$ be linearly independent roots with $w=s_{\alpha_{1}} s_{\alpha_{2}} \ldots s_{\alpha_{k}}$. By $Q$ we denote the sublattice generated by $\alpha_{1}, \alpha_{2}, \ldots, \alpha_{k} \in R_{r}$.

Lemma 1.14. There holds $Q=T\left(Q^{*}\right)$.

Proof. Set $w_{j}=s_{\alpha_{1}} s_{\alpha_{2}} \cdots s_{\alpha_{j}}$. By induction on $k$ we can show the equality

$$
w(x)=x+\sum_{j=1}^{k}\left(x, \alpha_{j}\right) w_{j-1}\left(\alpha_{j}\right) .
$$


Note, moreover, that $w_{j-1}\left(\alpha_{j}\right)(1 \leq j \leq k)$ is a basis of $Q$ since $w_{j-1}\left(\alpha_{j}\right)=\alpha_{j}+$ (a linear combination of $\alpha_{1}, \ldots, \alpha_{j-1}$ ). Choose an arbitrary $y \in Q$. We have integers $n_{1}, \ldots, n_{k}$ with $y=\sum_{j=1}^{k} n_{j} w_{j-1}\left(\alpha_{j}\right)$. By definition of the dual module we have an element $x \in Q^{*}$ with $\left(x, \alpha_{j}\right)=n_{j}$ for $1 \leq j \leq k$. By (1) we have $y=-T(x)$, which implies $Q \subset T\left(Q^{*}\right)$. Moreover, for every $x \in Q^{*}$ we have $-T(x)=w(x)-x \in Q$ by $(1)$, since $\left(x, \alpha_{i}\right) \in \mathbf{Z}$ for every $i$. Thus, $Q \supset T\left(Q^{*}\right)$.

Lemma 1.15. Let $\xi \in M \otimes \mathbf{Q}$ be the element with $x=T(\xi)=(1-w) \xi$. We have

$$
\sum_{0 \leq a<b<m}\left(w^{a}(x), w^{b}(y)\right)=-m(\xi, y) .
$$

Proof. Set $P=\sum_{a=1}^{m-1}(m-a) w^{a}$. Since $\left(1-w^{-1}\right) P=-m+N$ with $N=\sum_{a=0}^{m-1} w^{a}$, and since $N(y)=0$, we have $\sum_{0 \leq a<b<m}\left(w^{a}(x), w^{b}(y)\right)=\left(x, \sum_{0 \leq a<b<m} w^{b-a}(y)\right)=$ $(x, P(y))=\left(\xi,\left(1-w^{-1}\right) P(y)\right)=(\xi,(-m+N)(y))=-m(\xi, y)$.

For a submodule $K$ of a free $\mathbf{Z}$-module $L$ the set $\{x \in L \mid n x \in K$ for some nonzero integer $n\}$ is called the primitive hull of $K$ in $L$, and is denoted by $\tilde{K}$. It is the minimum submodule containing $K$ such that the quotient $L / \tilde{K}$ is free.

Proposition 1.16. We have $M \supset Q \supset T\left(M^{*}\right)$ and $Q / T\left(M^{*}\right)$ corresponds to a maximal isotropic subgroup of $H^{1}\left(G, U_{r}\right) \cong M / T\left(M^{*}\right)$.

Proof. By Lemma $1.13(2)$ we have $M=\tilde{Q}$. Thus, one has $Q \subset M \subset M^{*} \subset Q^{*}$. By Lemma 1.15 one knows $T\left(M^{*}\right) \subset T\left(Q^{*}\right)=Q \subset M$. Choose any elements $\bar{x}, \bar{y} \in Q / T\left(M^{*}\right)$ and their representatives $x, y \in Q$. By Lemma 1.14 we can choose an element $\xi \in Q^{*}$ with $x=T(\xi)$. Recall that $\langle\bar{x}, \bar{y}\rangle$ is equal to the class of $\sum_{0 \leq a<b<m}\left(w^{a}(x), w^{b}(y)\right)=-m(\xi, y)$ in $\mathbf{Z}_{m}$ by Lemma 1.15. Since $(\xi, y) \in \mathbf{Z}$, one has $\langle\bar{x}, \bar{y}\rangle=0$. Thus, $Q / T\left(M^{*}\right)$ defines an isotropic subgroup. Besides, since

$$
\left[M: T\left(M^{*}\right)\right]=[M: Q]\left[T\left(Q^{*}\right): T\left(M^{*}\right)\right]=[M: Q]\left[Q^{*}: M^{*}\right]=[M: Q]^{2},
$$

it is maximal by Lemma 1.11 .

Corollary 1.17. For any cyclic subgroup $G$ of $W\left(R_{r}\right)$ with a generator $w$, choose linearly independent roots $\alpha_{1}, \alpha_{2}, \ldots, \alpha_{k} \in R_{r}$ with $w=s_{\alpha_{1}} s_{\alpha_{2}} \cdots s_{\alpha_{k}}$. Let $Q$ be the sublattice generated by $\alpha_{1}, \alpha_{2}, \ldots, \alpha_{k} \in R_{r}$ and $M$ be the primitive hull of $Q$ in $U_{r}$. Then we have an exact sequence of abelian groups

$$
0 \rightarrow M / Q \rightarrow H^{1}\left(G, U_{r}\right) \rightarrow M / Q \rightarrow 0 .
$$

In particular, $h^{1}=[M: Q]^{2}$, where $h^{1}$ denotes the order of the group $H^{1}\left(G, U_{r}\right)$.

Remark. Now it is obvious that the Brauer group of a rational surface over a finite field always carries a canonical nondegenerate alternating form. Thus, we can improve a result in Milne [12]. Indeed, let $Y$ be a surface defined over a finite field $k$, and $\bar{k}$ be the algebraic closure of $k$. We assume that the pull-back $X=Y \otimes \bar{k}$ is 
a projective irreducible smooth rational surface over $\bar{k}$. Here $X$ is not necessarily a Del Pezzo surface. The Galois group Gal $=\operatorname{Gal}(\bar{k} / k)$ is isomorphic to the profinite completion $\hat{\mathbf{Z}}$ of $\mathbf{Z}$, and $H^{p}\left(\mathrm{Gal}, \bar{k}^{*}\right)=0$ for $p>0$. The lattice $U=$ $\mathrm{NS}(X)=\operatorname{Pic}(X)$ is a unimodular lattice, and the image $G$ of the homomorphism Gal $\rightarrow \operatorname{Aut}(U)$ is a cyclic group. Since $H^{2}\left(X_{e t}, \mathbb{G}_{m}\right)=0$ (Milne [12]), we have $\operatorname{Br}(Y) \cong H^{1}(\operatorname{Gal}, \operatorname{Pic}(X)) \cong H^{1}(G, U)$. Since the action of $G$ fixes the canonical class $\omega \in U$ and since $(x, x)$ is an even integer for every $x \in U$ with $(\omega, x)=0$, we can show that the canonical bilinear form on $\operatorname{Br}(Y)$ is alternating by the same reasoning as in Theorem 1.10.

\section{Comments on the tables}

Table 1 and Table 2 in the Supplement section give some information of the conjugacy classes of the Weyl group $W\left(E_{7}\right)$ and $W\left(E_{8}\right)$ and their action on the lattice $U_{7}$ and $U_{8}$ respectively. The classification of conjugacy classes is due to Carter [4].

(1) The first column gives the number of the class of conjugate elements.

(2) The second column gives the Carter symbol, a symbol to distinguish conjugacy classes due to Carter. For every $w \in W\left(E_{r}\right)$ with $r=6,7$ or 8 we have linearly independent roots $\alpha_{1}, \alpha_{2}, \ldots, \alpha_{k} \in R_{r}$ with $w=s_{\alpha_{1}} s_{\alpha_{2}} \cdots s_{\alpha_{k}}$. By Carter [4] we can assume, moreover, $\left(\alpha_{i}, \alpha_{j}\right)=0$ if $i \equiv j(\bmod 2)$ and $i \neq j$. With roots $\alpha_{1}, \alpha_{2}, \ldots, \alpha_{k} \in R_{r}$ satisfying these conditions we can associate a graph $\Gamma$ (i.e., a finite 1-dimensional complex) by the following rules:

(i) The set of vertices of $\Gamma$ has one-to-one correspondence with the set $\left\{\alpha_{1}, \alpha_{2}\right.$, $\left.\ldots, \alpha_{k}\right\}$.

(ii) The vertex corresponding to $\alpha_{i}$ and the vertex corresponding to $\alpha_{j}(i \neq j)$ is connected in $\Gamma$ by an edge if and only if $\left(\alpha_{i}, \alpha_{j}\right) \neq 0$.

The graph $\Gamma$ can contain cycles with an even number of vertices. If it contains no cycles, it coincides with the Dynkin graph of the root system generated by $\left\{\alpha_{1}, \alpha_{2}, \ldots, \alpha_{k}\right\}$ or with the Dynkin graph of the lattice generated by $\left\{\alpha_{1}, \alpha_{2}\right.$, $\left.\ldots, \alpha_{k}\right\}$. Each possible type of connected components of $\Gamma$ has an associated symbol $\Delta$ by Carter. The Carter symbol of $\Gamma$ is the formal sum $\sum_{i} m_{i} \Delta_{i}$ with the number $m_{i}$ of connected components in $\Gamma$ corresponding to the symbol $\Delta_{i}$. We can use Carter symbols to denote the conjugacy class, since conjugate elements give the same graph. Note here that sometimes nonconjugate elements give the same graph. In this case symbols $(\cdot)^{\prime}$ and $(\cdot)^{\prime \prime}$ are used to distinguish between them. (See last Remark in this section.)

(3) The third column gives the value of the index of an element $w \in W\left(E_{r}\right)$ in the conjugacy class. The index is defined in $[11$, p. $152, \S 28.2]$ and plays an important role in Manin's theory. First we define the set of exceptional vectors by

$$
I_{r}=\left\{\lambda \in U_{r} \mid(\lambda, \lambda)=-1,(\omega, \lambda)=-1\right\} .
$$

We consider an element $w \in W\left(R_{r}\right)$. A set $\left\{\lambda_{1}, \lambda_{2}, \ldots, \lambda_{k}\right\} \subset I_{r}$ is called $w$ exceptional if:

(i) the set $\left\{\lambda_{1}, \lambda_{2}, \ldots, \lambda_{k}\right\}$ is $w$-invariant;

(ii) $\left(\lambda_{i}, \lambda_{j}\right)=0$ for $i \neq j$.

The index $i(w)$ is defined to be the maximal number of elements of $w$-exceptional sets. 
(4) The fourth column (Measure ${ }^{-1}$ ) gives the quotient of the order of the group and the number of elements in the conjugacy class.

(5) Frame symbols in the fifth column describe the characteristic polynomial $\psi(t)$ of the homomorphism $w: U_{r} \rightarrow U_{r}$ associated with an element $w \in W\left(R_{r}\right)$ in the class (Frame [8]). If the Frame symbol is $\prod_{m} m^{n_{m}}$, then it implies that $\psi(t)=\prod_{m}\left(t^{m}-1\right)^{n_{m}}$. In particular, $n_{1}$ equals the trace of $w$ on $U_{r}$, and the order of $w$ is equal to the least common multiple of integers $\left\{m \mid n_{m} \neq 0\right\}$. If the base field $k$ is a finite field with $q$ elements, then the number $N$ of $k$-points on the corresponding surface is given by $N=q^{2}+q n_{1}+1$ ([11, p. 143, Theorem $27.1])$. Note that $\psi(t)$ is different from the characteristic polynomial $\psi_{0}(t)$ of the homomorphism induced by $w$ on the orthogonal complement of the canonical class $\omega \in U_{r}$. Obviously, the two polynomials satisfy $\psi(t)=(t-1) \psi_{0}(t)$. Moreover, note that Frame symbols are different from symbols in the table on pp. 176-177 of Manin [11].

(6) Let $\alpha_{1}, \alpha_{2}, \ldots, \alpha_{k} \in R_{r}$ be roots satisfying the conditions in (2) above. By $Q$ we denote the sublattice generated by $\alpha_{1}, \alpha_{2}, \ldots, \alpha_{k} \in R_{r}$. A lattice generated by a set of roots is classified by the associated Dynkin graph. The sixth column gives the associated Dynkin graph with $Q$. If the Dynkin graph is $\sum a_{k} A_{k}+\sum b_{\ell} D_{\ell}+\sum c_{m} E_{m}$, then this implies that $Q \cong \sum Q\left(A_{k}\right)^{a_{k}}+\sum Q\left(D_{\ell}\right)^{b_{\ell}}+$ $\sum Q\left(E_{m}\right)^{c_{m}}$ (orthogonal direct sum), where for a connected Dynkin graph $\Gamma_{0}=$ $A_{k}, D_{\ell}, E_{m}, Q\left(\Gamma_{0}\right)$ denotes the root lattice associated with $\Gamma_{0}$. By Carter's theory we can easily determine the Dynkin graph of $Q$.

(7) Let $M$ be the primitive hull of the above $Q$ in $U_{r}$. It turns out that $M$ is also generated by roots. The Dynkin graph associated with $M$ is given in the seventh column. By the theory of Dynkin (Coxeter [5], Dynkin [7]) we can easily determine $M$ and $M / Q$.

(8) The eighth column contains the values of $H^{1}\left(G, U_{r}\right)$, where $G$ is the cyclic group in $W\left(R_{r}\right)=W\left(E_{r}\right)$ generated by an element in the conjugacy class. By the results in $\S 1$ the order $h^{1}$ is equal to $[M: Q]^{2}=\operatorname{det} Q / \operatorname{det} M$, and it is easy to calculate it. On the other hand, by the property of the group cohomology, $m x=0$ for every $x \in H^{1}\left(G, U_{r}\right)$, if $m$ is the order of the group $G$. Therefore, we see that the group $H^{1}\left(G, U_{r}\right)$ is uniquely determined by the order $h^{1}$ if for every prime number $p$ dividing $h^{1}, p^{2}$ does not divide $m$. Even if the last condition is not satisfied, except in a few cases we can determine the abelian group $H^{1}\left(G, U_{r}\right)$ by Corollary 1.12 and Corollary 1.17. The exceptions are the following three cases (all are in the case of $\left.W\left(R_{8}\right)=W\left(E_{8}\right)\right)$ : No.1 $A_{7}+A_{1}$, No.10 $2 A_{3}+2 A_{1}$, No.17 $2 D_{4}\left(a_{1}\right)$. For these three exceptions we apply Proposition $1.9, H^{1}\left(G, U_{r}\right) \cong M /(1-w) M^{*}$, and determine the group structure.

(9) In the ninth column, the symbol $a^{r} b^{s} \ldots$ means that there are $r$ orbits of order $a, s$ orbits of order $b$, etc. A notation of the type $2^{4} \cdot 2^{24}$ means that, of 28 orbits of 2 exceptional vectors, four have one type of the characteristic sequence and twenty-four another. The characteristic sequence of an orbit $\lambda_{1}, \lambda_{2}, \ldots, \lambda_{k} \subset I_{r}$ $\left(\lambda_{i}=w^{i-1}\left(\lambda_{1}\right)\right.$ for $2 \leq i \leq k$, and $\left.\lambda_{1}=w^{n}\left(\lambda_{1}\right) \Leftrightarrow n \equiv 0 \quad(\bmod k)\right)$ of a cyclic group $G$ with a generator $w$ is the sequence $\left(\left(\lambda_{1}, \lambda_{2}\right),\left(\lambda_{1}, \lambda_{3}\right), \ldots,\left(\lambda_{1}, \lambda_{\ell}\right)\right)$ where $\ell=[k / 2]$ (the Gauss symbol) by definition. Note that the intersection matrix $\left(\left(\lambda_{i}, \lambda_{j}\right)\right)$ is uniquely determined by the characteristic sequence.

(10) The tenth column in the case $W\left(R_{7}\right)=W\left(E_{7}\right)$ shows the conjugacy classes 
which we cannot distinguish by the information in the ninth column.

Remark. We can find some links among the table for $E_{6}$ in Manin [11, pp. 176-177] and our tables below for $E_{7}$ and $E_{8}$, if we make the correction in Manin's table.

First, there are natural mappings among tables induced by blowing-up of a point on the corresponding surface. Let $f$ be the mapping from the set of rows of the table for $E_{7}$ to the set of rows of the table for $E_{8}$ satisfying $f(1)=98, f(i)=i+36$ for $2 \leq i \leq 24, f(i)=i+41$ for $25 \leq i \leq 33, f(34)=76, f(35)=79, f(36)=80$, $f(37)=109, f(38)=87, f(39)=88, f(40)=95, f(41)=101, f(42)=103$, $f(43)=104, f(44)=107, f(45)=110, f(i)=i+45$ for $46 \leq i \leq 52, f(i)=i+46$ for $53 \leq i \leq 56, f(i)=i+48$ for $57 \leq i \leq 59$ and $f(60)=111$. This $f$ has the following properties:

1. It is almost injective. Indeed, if we exclude rows $40,41,44,50,55$ and 59 (for them the Carter symbol has $(\cdot)^{\prime}$ or $\left.(\cdot)^{\prime \prime}\right)$ from the table for $E_{7}$, then it becomes injective. Besides, $f(40)=f(50)=95, f(41)=f(55)=101$ and $f(44)=f(59)=107$.

2. It almost preserves Carter symbols. Indeed, if we ignore symbols $(\cdot)^{\prime}$ and $(\cdot)^{\prime \prime}$, then the Carter symbol of the row $i$ and that of $f(i)$ are equal.

3. The index of the row $f(i)$ is greater than or equal to 1 plus the index of the row $i$. Moreover, except for rows 1 and 37 through 45, the equality always holds.

4. If the Frame symbol of the row $i$ is $\prod_{m} m^{n_{m}}$, then the Frame symbol of the row $f(i)$ is equal to $1^{n_{1}+1} \cdot \prod_{m \neq 1} m^{n_{m}}$.

5. For items in the three columns $Q, M$ and $H^{1}$, the item of the row $i$ and the item of the row $f(i)$ are equal ([11, p. 155, Theorem 29.1, Lemma 29.1.2]).

6 . For items in the remaining two columns "Measure ${ }^{-1}$ " and "Number of orbits" we can find some relations between the corresponding rows.

Also, there exists an injective mapping with similar properties from the set of rows of the corrected Manin's table for $E_{6}$ to the set of rows of our table for $E_{7}$.

Second, for rows with positive index we can define the correspondence in the opposite direction, which is induced by blowing-down of exceptional curves on the surface. For rows with index 1 it gives the inverse mapping of the above $f$. It preserves the structure of $H^{1}$. However, it depends on the choice of the blowingdown. It is tiresome to give a clear description for this correspondence if the index is greater than 1 , so we do not give it here ([11, p. 153, Theorem 28.3 and $\S 28.4])$.

Remark. We here give a remark on Carter symbols with $(\cdot)^{\prime}$ or $(\cdot)^{\prime \prime}$ in the second column.

Carter in [4] does not explain the distinction between $(\cdot)^{\prime}$ and $(\cdot)^{\prime \prime}$. Since by data in the table in [4] we can distinguish between them, perhaps he thought that no explanation was necessary. We here would like to note that similar symbols were used in Dynkin [7]. By the nature of Carter's theory, after excluding items whose Carter's graph contains a cycle, Carter's list has to coincide with Dynkin's list in [7] of conjugacy classes of root subsystems in the root system $E_{7}$ or $E_{8}$. Thus perhaps Carter used these symbols following Dynkin. Since any items in the second column with $(\cdot)^{\prime}$ or $(\cdot)^{\prime \prime}$ has Carter's graph without a cycle, items with these symbols are in a one-to-one correspondence with items in Dynkin's list with $[\cdot]^{\prime}$ or $[\cdot]^{\prime \prime}$.

Note that Dynkin gave an explanation on the distinction between $[\cdot]^{\prime}$ and $[\cdot]^{\prime \prime}$ in 
a footnote in [7]. According to him "The symbols $[\cdot]^{\prime}$ and $[\cdot]^{\prime \prime}$ have the following meaning: In the case of the subalgebra $E_{7}$, the subalgebras indicated with one accent are contained in the subalgebra $A_{7}$, and those with two accents are not contained in $A_{7}$; in the case of the algebra $E_{8}$, the subalgebras with one accent are contained in $A_{8}$, and those with two accents are not contained in $A_{8} . "$

Here we notice that their usage of symbols is just opposite. Carter's item with $(\cdot)^{\prime}$ corresponds to Dynkin's item with $[\cdot]^{\prime \prime}$, and Carter's with $(\cdot)^{\prime \prime}$ to Dynkin's with $[\cdot]^{\prime}$ for both cases $W\left(R_{7}\right)=W\left(E_{7}\right)$ and $W\left(R_{8}\right)=W\left(E_{8}\right)$.

\section{ACKNOWLEDGMENTS}

I express thanks to Jun-ichi Matsuzawa for informing me of the classical result of E. Cartan [3], and to Alexander V. Sardo-Infirri for correcting my English in the manuscript. Also I am indebted to the inventors of the Macintosh computer and the developers of the excellent pieces of software "Mathematica" and "Excel".

\section{REFERENCES}

1. N. Bourbaki, Groupes et algèbre de Lie, Chaps. 4-6, Hermann, Paris, 1968.

2. H. Cartan and S. Eilenberg, Homological algebra, Princeton Univ. Press, Princeton, NJ, 1956. MR 17:1040e

3. E. Cartan, Sur la réduction à sa forme canonique de la structure d'un groupe de transformations fini et continu, Amer. J. Math. 18 (1896), 1-61.

4. R. W. Carter, Conjugacy classes in the Weyl group, Compositio Math. 25 (1972), 1-59. MR 47:6884

5. H. S. M. Coxeter, Finite groups generated by reflections and their subgroups generated by reflections, Proc. Cambridge Philos. Soc. 30 (1934), 466-482.

6. Patric Du Val, On isolated singularities of surfaces which do not affect the conditions of adjunction, (Part II), Proc. Cambridge Philos. Soc. 30 (1934), 460-465.

7. E. B. Dynkin, Semisimple subalgebras of semisimple Lie groups, Amer. Math. Soc. Transl. (2) 6 (1957), 111-244. MR 13:904

8. J. S. Frame, The classes and representations of the groups of 27 lines and 28 bitangents, Ann. Math. Pura Appl. (4) 32 (1951), 83-119. MR 13:817i

9. B. È. Kunyavskii, A. N. Skorobogatov, and M. A. Tsfasman, Del Pezzo surfaces of degree four, Mém. Soc. Math. France (N. S.) 37, Supplément au Bulletin de la S. M. F., vol. 117, Fasc. 2, 1989, pp. 1-113. MR 90k:14035

10. Yu. I. Manin, Rational surfaces over perfect fields I, Inst. Hautes Etudes Sci. Publ. Math. 30 (1966), 55-113. MR 37:1373

11. L Cubic forms, 2nd ed., North-Holland, Amsterdam, New York, Oxford, 1986. MR 87d:11037

12. J. S. Milne, The Brauer group of a rational surface, Invent. Math. 11 (1970), 304-307. MR 44:2756

13. Étale cohomology, Princeton Univ. Press, Princeton, NJ, 1980. MR 81j:14002

14. P. Swinnerton-Dyer, The Brauer group of cubic surfaces, Math. Proc. Cambridge Philos. Soc. 113 (1993), 449-460. MR 94a:14038

15. T. Urabe, maninh1, Mathematica notebooks for calculation of Del Pezzo surfaces of degree 2 or 1, anonymous ftp-able, /tnt/maninh1-mac.tar.Z, ftp.math.metro-u.ac.jp (1994).

16. S. Wolfram, Mathematica, A system for doing mathematics by computer, Addison-Wesley, Redwood City, 1988.

17. Yu. G. Zarkhin, The Brauer group of an Abelian variety over a finite field, Math. USSR-Izv. 20, No. 2 (1983), 203-234. MR 83h:14035

Department of Mathematics, Tokyo Metropolitan University, Minami-Ohsawa 1-1, HACHIOJI-SHI, TOKYO, 192-03 JAPAN

E-mail address: urabe@math.metro-u.ac.jp 